\title{
Prevention of Complications in Endoscopic Third Ventriculostomy
}

\author{
Tae-Young Jung, M.D., Ph.D., ${ }^{*}$ Sangjoon Chong, M.D., ${ }^{2 *}$ In-Young Kim, M.D., Ph.D., Ji Yeoun Lee, M.D., Ph.D., ${ }^{2,3}$ \\ Ji Hoon Phi, M.D., Ph.D., ${ }^{2}$ Seung-Ki Kim, M.D., Ph.D., ${ }^{2}$ Jae-Hyoo Kim, M.D., Ph.D., ${ }^{4}$ Kyu-Chang Wang, M.D., Ph.D. ${ }^{2}$ \\ Department of Neurosurgery, Chonnam National University Medical School and Hwasun Hospital, Hwasun, Korea \\ Division of Pediatric Neurosurgery and Clinical Pediatric Neuroscience Center, ${ }^{2}$ Seoul National University Children's Hospital, Seoul, Korea \\ Department of Anatomy, ${ }^{3}$ Seoul National University College of Medicine, Seoul, Korea \\ Department of Neurosurgery, ${ }^{4}$ Chonnam National University Medical School and Hospital, Gwangju, Korea
}

A variety of complications in endoscopic third ventriculostomy have been reported, including neurovascular injury, hemodynamic alterations, endocrinologic abnormalities, electrolyte imbalances, cerebrospinal fluid leakage, fever and infection. Even though most complications are transient, the overall rate of permanent morbidity is $2.38 \%$ and the overall mortality rate is $0.28 \%$. To avoid these serious complications, we should keep in mind potential complications and how to prevent them. Proper decisions with regard to surgical indication, choice of endoscopic entry and trajectory, careful endoscopic procedures with anatomic orientation, bleeding control and tight closure are emphasized for the prevention of complications.

Key Words : Neuroendoscopy · Ventriculostomy · Peoperative complications/prevention \& control.

\section{INTRODUCTION}

Although endoscopic third ventriculostomy (ETV) is a safe procedure, a variety of complications have been reported, mostly related with the surgical procedure. The overall morbidity rate reported is $8.5 \%$, ranging from 0 to $31.2 \%$, and the overall rate of permanent morbidity is $2.38 \%{ }^{6,7,19,25)}$. Even though lethal complications have been rarely reported, the overall mortality rate is $0.28 \%$, and as high as $1.28 \%$. Causes of mortality include thalamic injury, basilar artery injury, meningitis, and sepsis.

Ventricular anatomy is altered in many diseases. The altered anatomy interferes with the correct placement of the fenestration on the third ventricle floor, which can cause vascular and neurological injuries and may be associated with significant complications and morbidity. In cases of developmental

- Received : January 25, 2017 •Revised : February 19, 2017 •Accepted : February 21, 2017

- Address for reprints : Kyu-Chang Wang, M.D., Ph.D. (Corresponder during the process of review and publication)

Division of Pediatric Neurosurgery and Clinical Pediatric Neuroscience Center, Seoul National University Children's Hospital, 101 Daehak-ro, Jongno-gu, Seoul 03080, Korea Tel : +82-2-2072-3489, Fax : +82-2-2072-0274, E-mail : kcwang@snu.ac.kr

Jae-Hyoo Kim, M.D., Ph.D. (Co-corresponding author)

Department of Neurosurgery, Chonnam National University Medical School and Hospital, 160 Baekseo-ro, Dong-gu, Gwangju 61469, Korea

Tel : +82-62-220-6600, Fax : +82-62-224-9865, E-mail : jaehyoo@chonnam.ac.kr

*These authors contributed equally to this work.

This is an Open Access article distributed under the terms of the Creative Commons Attribution Non-Commercial License (http://creativecommons.org/licenses/by-nc/4.0) which permits unrestricted non-commercial use, distribution, and reproduction in any medium, provided the original work is properly cited. 
anomalies such as myelomeningocele and multilocular hydrocephalus, it is not easy to identify the transparent membrane in front of the mammillary bodies ${ }^{19,25)}$. Basal ganglia and large thalamic tumors cause significant ventricular distortion ${ }^{24)}$. In older patients or those with previous cerebrospinal fluid (CSF) infection, the third ventricle floor may be thickened, and the basilar artery is not easily identified ${ }^{7)}$. In some individual patients, there is a very narrow space between the clivus and the mammillary bodies or basilar artery. Moreover, repeated ETV is associated with significantly increased complication risk $^{18,25)}$.

The complications of ETV have been underestimated. Various types of complications have been reported, including cerebral herniation syndromes, cardiac arrhythmias, injury to the hypothalamic-pituitary axis and/or structures near the floor of the third ventricles such as the cranial nerves and basilar artery, injury of large ependymal veins, meningitis, sepsis, and cognitive dysfunction ${ }^{6,7,22,25)}$. This article reviews various types of complications and discusses how to prevent these serious complications.

\section{COMPLICATIONS OF ENDOSCOPIC THIRD VENTRICULOSTOMY}

\section{Vascular injury}

Minor bleeding occurs in up to $16.5 \%$ of cases ${ }^{7,19)}$. Bleeding occurs with accidental tearing or unusual stretching of the veins by the endoscopic devices. Small cortical, parenchymal or ependymal vessels may be torn. The majority of bleeding can be controlled using continuous irrigation with wash-out and generous compression ${ }^{28)}$. Major bleeding is reported in $0.49 \%$ of cases due to injury to branches of the basilar artery or septal and thalamostriate veins ${ }^{19)}$. Injury to the basilar artery or basilar perforating arteries is the most feared intraoperative complication, occurring in $0.2-0.3 \%$ of cases and associated with high mortality and morbidity ${ }^{6,22)}$. The tortuosity and herniation of the basilar complex against the floor of the third ventricle or an opaque neural floor can elevate the risk of this complication ${ }^{12)}$.

\section{Injury of neural structures}

Cerebral herniation syndrome occurs because of closed and obstructed outflow channels or excessive irrigation ${ }^{25)}$. The volume of irrigation and intracranial pressure (ICP) changes should be kept in mind. Significantly increased ICP causes delayed awakening and persistent confusion. Intraoperative injury of neural structures affects the fornix during passage through the foramen of Monro, the thalamus by direct injury, the hypothalamus due to eccentric location of the ostomy, and the oculomotor nerve upon penetration of the third ventricle floor $^{7,22)}$.

Reported rates of transient neurologic symptoms vary. Transient ocular divergence and anisocoria can occur from stretching of the midbrain and third or sixth cranial nerves palsies. Inadvertent brain injury causes transient loss of consciousness, neurological deficits, confusion, memory loss, convulsions and impaired cognitive function. Among such cases, the rate of permanent neurological morbidity was estimated at $0.5-1.4 \%{ }^{6,7,22)}$.

\section{Hemodynamic alterations}

Intraoperative changes in heart rate, rhythm and mean arterial pressure can be recorded during the procedure by anesthesiologists. Hemodynamic changes are frequent and mostly transient ${ }^{19)}$. Surgeons should be alerted to hemodynamic changes during ETV procedures. When tachycardia, bradycardia, hypertension, hypotension, and arrhythmia are noticed, surgeons should pause fluid irrigation, ballooning and advance of endoscopy. Rarely, intraoperative cardiac arrest and uncontrollable postoperative high-frequency tachypnea have been reported ${ }^{4,14)}$.

Cardiovascular responses may be induced by a sudden rise in ICP secondary to high-speed irrigation or obstruction of the outflow of fluid, which can lead to impaired cerebral perfusion. Tachycardia and hypertension are associated with increased ICP. Inadvertent stimulation or injury to the posterior hypothalamus or third cranial nerve can also induce hemodynamic changes. The reported rate of bradycardia at the time of fenestration of the third ventricle floor is $26.8-43 \%{ }^{3,19)}$.

\section{Endocrine abnormalities and electrolyte imbal- ances}

The overall rate of hormonal morbidity is $0.94 \%{ }^{6,7)}$. The tuber cinereum is a part of the hypothalamic-pituitary axis of cerebral endocrine regulation. Hormonal morbidity mainly 
includes transient diabetes insipidus of hypothalamic origin, which remains permanent in some patients. Opening close to the infundibular recess resulted in permanent diabetes insipidus and transient loss of thirst ${ }^{25}$. Diabetes insipidus was reported in $0.64 \%$ of cases, weight gain in $0.27 \%$, and precocious puberty in $0.04 \%{ }^{6}$. Additionally, amenorrhea and partial growth hormone deficiency were reported due to hypothalamic injury ${ }^{1726)}$.

Electrolyte disorders such as hyponatremia, hypernatremia and hyperkalemia can occur and in most cases reverse spontaneously ${ }^{2,6,19)}$. This may be explained by a transient hormonal disturbance related to the hypothalamus, which is situated in the third ventricle floor. Interestingly, postoperative transient isolated hyperkalemia without changes in the serum sodium level was reported 3-4 days after surgery ${ }^{2}$.

\section{CSF leakage, fever and infection}

The reported rate of CSF leaks was $1.7 \%$ overall, with reports as high as $5.2 \%{ }^{6,7)}$. This is often the first sign of early failure, and persistent CSF leakage can be a sign of treatment failure. This is treated by resuturing of the skin incision, and serial lumbar CSF drainage if increased ICP is suspected.

Postoperative fever was reported in $65.1 \%$ to $84.4 \%$ of cases, with especially high rates among children ${ }^{11,21)}$. Fever may be caused by hypothalamic irritation due to mechanical injury, small hemorrhages or air in the ventricle. Fever frequently develops after procedures, peaking the day of surgery and/or rapidly subsiding, usually within 3 days. In cases of sustained fever, increased temperature 48 hours postoperatively and clinical illness, various infections including meningitis or ventriculitis should be considered.

Infections such as meningitis or ventriculitis were reported in $1.81 \%$ to $6.1 \%$ of cases in the immediate postoperative peri$\mathrm{od}^{6,7)}$. Infectious complications are commonly associated with CSF leakage. Although antibiotic treatment is usually effective, progression to sepsis was reported in rare cases.

\section{PREVENTION OF COMPLICATIONS}

We would like to emphasize preventive measures for several of the complications mentioned above.

\section{Proper surgical indication}

The surgical risk of neural injury in ETV is higher than that of shunting. Unnecessary trials of ETV should be avoided as much as possible. Prediction of success after ETV is clinically significant for the prevention of complications. The prediction of success is discussed in another chapter.

In this chapter, we address an additional aspect of post-ETV aggravation of ventriculomegaly. In cases when craniectomy or craniotomy, especially cranial remodeling, is combined with ETV either simultaneously or in a staged fashion, ETV should be reconsidered. In contrast to shunting surgery, it is not rare for ETV to require pressures higher than normal ICP to work normally. The ventricles are frequently larger than normal even in the post-ETV 'well-functioning' state. When craniotomy is combined with ETV in patients with hydrocephalus, the relatively high ICP may elevate the craniotomy flaps and lead to progressive ventriculomegaly. If the craniotomy flap is loosely fixed or easily expands, the ICP does not reach the threshold of CSF absorption and the ventricles may dilate even if the ICP is lower than in the preoperative state. Therefore, rigid fixation of craniotomy flaps is required. Mildly increased ICP caused by rigid fixation pushes the CSF through the absorption sites, such as arachnoid granulations, preventing progression of hydrocephalus and leading to successful ETV. If the ventricle size is stabilized at a non-physiologically high ICP, the ETV procedure should be regarded as a failure.

\section{Point of ventricular entry}

For typical ETV, the most preferable site of entry is around the coronal suture, approximately $3 \mathrm{~cm}$ laterally from the midline in adults. However, this should be modified according to the shape of ventricles and age of the patient.

When ETV and an additional procedure such as biopsy or septostomy is required at the same time, the site of entry and the shape of the burr hole should be modified. For example, a pineal mass with associated hydrocephalus can be managed by ETV and simultaneous biopsy. In this case, we prefer a burr hole placed close to the hairline with anteroposteriorly elongated shape. The endoscope is introduced along the angle between the usual ETV entry site and the pineal biopsy site (Fig. 1). Neuro-navigation can be helpful to select the entry point and trajectory ${ }^{20)}$. Because of possible bleeding from the tumor, we 


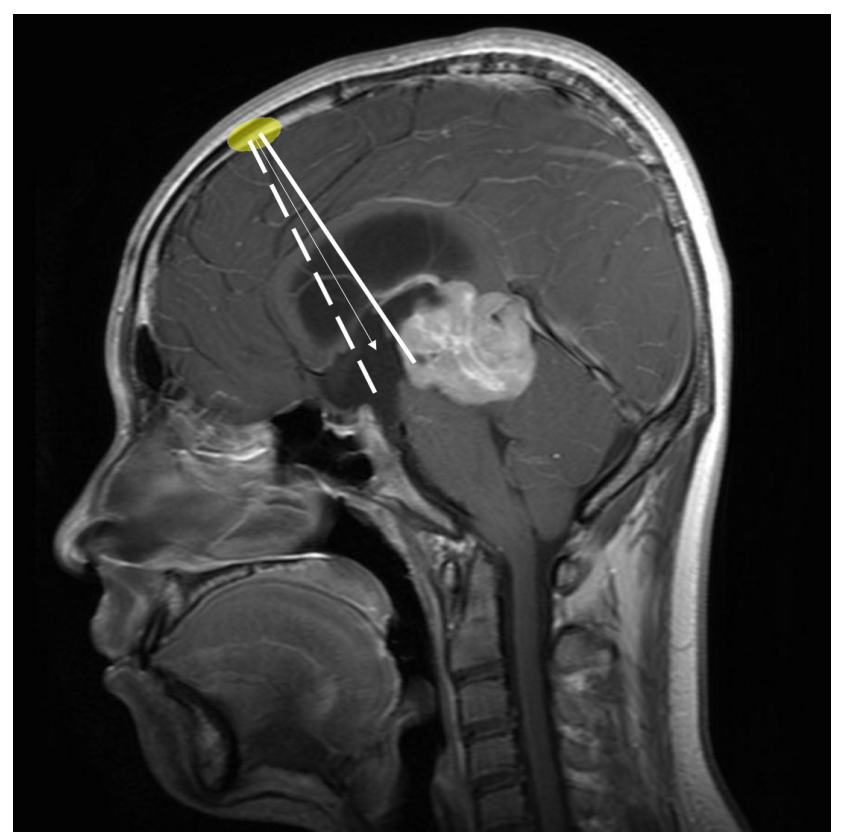

Fig. 1. A recommendable trajectory for simultaneous ETV and biopsy is shown as an arrow on the midsagittal MR image in a patient with a pineal mass and hydrocephalus. It is set between two paths for endoscopic third ventriculostomy (dotted line) and biopsy (solid line). Note that the burr hole (colored ellipse) is elongated in an anteriorposterior direction. ETV : endoscopic third ventriculostomy, MR : magnetic resonance.

perform the ETV first, then perform the biopsy later. The clots are aspirated around the stoma of the ETV if present.

\section{Insertion of the endoscope}

For the puncture of the ventricle with an endoscope, exact measurement of the ventricle system is important to avoid unnecessary injury to the brain. Although endoscopes have become smaller in diameter compared to previous eras, they are still much larger than a shunt catheter and could cause significant brain damage with neurological deficits if placed in the wrong direction.

Image-guided neuro-navigation or intraoperative sonography are excellent tools to assist in ventricular access, especially when the size of the ventricle is relatively small. However, for moderate- or large-size ventricles, careful aiming of the endoscope according to multi-directional neuroradiologic images is an acceptable method ${ }^{8)}$.

\section{Anatomical orientation for steering of endo- scopes}

The obstacles to a successful ETV after entering into the ventricle are disorientation and bleeding.

Sometimes the endoscope is inserted too anteriorly into the frontal horn, where the choroid plexus is absent. The endoscope should be steered to the posterior side to find the choroid plexus at the foramen of Monro.

It is not rare to encounter a septal defect when the hydrocephalus is congenital in origin or long-lasting. It is not difficult to make a proper orientation by identifying the relative positions of anatomical structures, such as the foramen of Monro, septal veins, thalamostriate veins and the choroid plexus.

For a typical ETV, the landmarks-foramen of Monro, septal vein, thalamostriate veins, mammillary bodies and infundibular recess-are prominent and disorientation is not a problem. Usually, the dorsum sellae and the top of the basilar artery are visible through the thinned floor of the third ventricle. Nonetheless, chronic inflammation or congenital anomalies, such as hydrocephalus in a myelomeningocele patient, can obscure the anatomy $y^{5,10,23,24)}$. Careful inspection of sagittal magnetic resonance images is helpful to determine the relationship between the basilar artery and the floor of the third ventricle, considering individual anatomical variations ${ }^{15}$. Again, intraoperative neuro-navigation is useful. Palpation of the dorsum sellae is another method but still presents some risk.

\section{Control of bleeding}

Sizable arteries are not exposed to the ventricle system. Only bleeding from the choroid plexus, veins or capillaries may be encountered during ventricular access in ETV. Therefore, gentle pressure is helpful and sufficient to control the bleeding because the bleeding pressure is not as high as in arteries.

Irrigation with saline is the most commonly used method for clearing the operative field and controlling bleeding. It is not reasonable to 'wash' a wound for hemostasis. Instead, compression is the 'common sense' approach for the control of bleeding. The 'washing' component of irrigation does not stop bleeding but the 'compression' exerted by the pressure from the fluid column of irrigation saline is helpful for the 
control of bleeding in endoscopy. Applying a water column with closure of the drainage port is a more effective way to manage the bleeding ${ }^{28)}$. However, water column tamponade at high pressure for longer than $4-5$ minutes may cause ischemic damage, especially when the patient is very young. Blood pressure and pulse rate should be carefully monitored during the period of water column tamponade. We apply intraoperative monitoring in relatively complex endoscopic procedures that usually require more frequent and longer tamponade, such as fenestration of deep-seated arachnoid cysts in young patients.

Although rare, cases of arterial injury around the ETV stoma are reported in the literature ${ }^{1,16,25)}$. In these cases, the tear of the arterial wall is small and massive saline irrigation is helpful. It takes a much longer time to control bleeding than in cases of bleeding from veins or capillaries. Careful followup is needed to detect the formation of pseudoaneurysms, which may require interventional neurovascular procedures.

\section{Insertion of instruments through the endoscopic port}

The visual range of an endoscope is less than $180^{\circ}$. The outlet at the ventricle side for endoscopic instruments is located just adjacent to the tip of the optic fiber. Therefore, the tip of the instrument is visible only when the instrument is advanced a short distance from the level of the tip of the optic fiber. If the instruments are inserted while the tip of the optic fiber is located too close to the wall of the ventricles, they may penetrate the wall of the ventricles before it is visualized through the endoscope. To prevent this kind of neurovascular injury, when instruments are inserted, the tip of the optic fiber should be oriented slightly away from the ventricular wall, staying in a wide space such as at the superior area of the lateral ventricle. In addition, the tip of instruments should be identified before they are advanced, proceeding as one piece with the optic fiber until they approximate the target (Fig. 2).

\section{Passing of an endoscope through the foramen of Monro}

Minor contusion injury to the fornix is common during the ETV procedure. It does not cause symptoms of memory disturbance because most injuries are mild and unilateral. However, if the injury is bilateral it may cause symptoms. Therefore, we do not recommend ETV through the contralateral

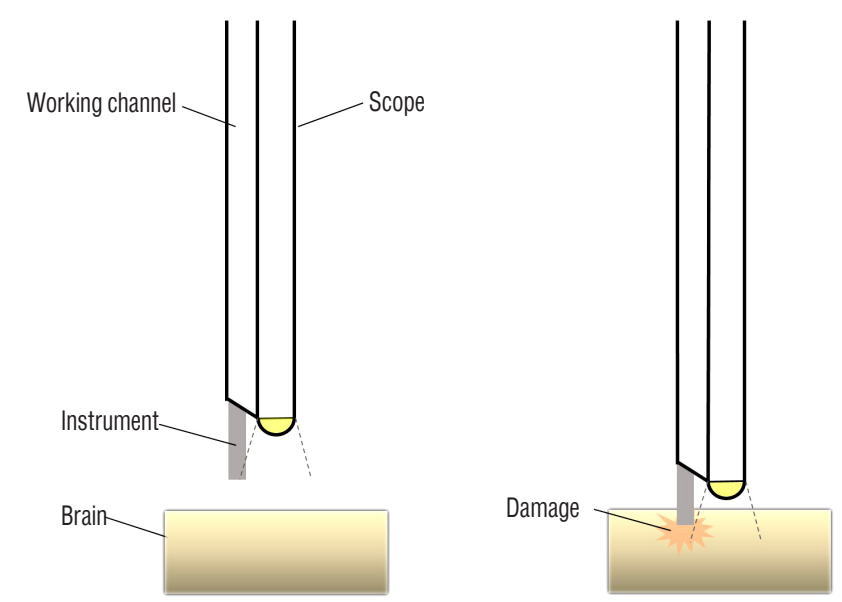

Fig. 2. A schematic drawing showing a case of brain damage caused by an instrument when the endoscope is too close to the ventricle wall before the instrument is identified through the endoscope (right). When the endoscope is placed sufficiently away from the wall (left), the instrument does not cause brain damage while it enters the endoscopic view.

foramen of Monro, especially if there was a significant injury to the fornix during a previous ETV.

If the foramen of Monro is small and occluded by choroid plexus, it is reasonable to coagulate the choroid plexus before the endoscope passes through the foramen. This may minimize bleeding from the injury to the choroid plexus. Applying moderate pressure with the fluid column of irrigation saline is a method to enlarge the foramen. This reduces the chance and degree of bleeding from the choroid plexus and injury to the fornix.

\section{Postoperative CSF leakage in infants}

It is well known that in infants, the failure rate of ETV is higher than in older children, probably due to poorer CSF absorption $^{9,13,27)}$. Furthermore, the layers of dura and scalp at the closure are thin and weak. Therefore, postoperative CSF leakage is one of the major concerns that leads to shunting surgery in infants. When we close the ETV wound in infants, we pay special attention to reinforcement of the burr hole site as well as plugging the tract using pieces of wedge-shaped absorbable gelatin sponge which are large enough not to fall into the ventricle. We make the best use of pericranial suture, dural sealants and a bone graft or a small metal piece to support the burr hole. 


\section{CONCLUSION}

Even though the complication rate of ETV is not so high, serious events should always be kept in mind. Understanding of potential surgical complications at each step, careful intraoperative monitoring and procedures, and close postoperative observation are necessary to prevent these complications.

\section{- Acknowledgements}

This research was supported by Basic Science Research Program through the National Research Foundation of Korea (NRF) funded by the Ministry of Science, ICT \& Future Planning (2015R1A2A1A15055781).

\section{References}

1. Abtin K, Thompson BG, Walker ML : Basilar artery perforation as a complication of endoscopic third ventriculostomy. Pediatr Neurosurg 28 : 35-41, 1998

2. Anandh B, Madhusudan Reddy KR, Mohanty A, Umamaheswara Rao GS, Chandramouli BA : Intraoperative bradycardia and postoperative hyperkalemia in patients undergoing endoscopic third ventriculostomy. Minim Invasive Neurosurg 45 : 154-157, 2002

3. Baykan N, Isbir O, Gercek A, Dagcnar A, Ozek MM : Ten years of experience with pediatric neuroendoscopic third ventriculostomy: features and perioperative complications of 210 cases. J Neurosurg Anesthesiol $17: 33-37,2005$

4. Bernard R, Vallee F, Mateo J, Marsella M, George B, Payen D, et al. : Uncontrollable high-frequency tachypnea: a rare and nearly fatal complication of endoscopic third ventriculostomy: case report and literature review. Minim Invasive Neurosurg 53 : 270-272, 2010

5. Bhagwati S, Mehta N, Shah S: Use of endoscopic third ventriculostomy in hydrocephalus of tubercular origin. Childs Nerv Syst 26 : 16751682,2010

6. Bouras T, Sgouros $S$ : Complications of endoscopic third ventriculostomy. J Neurosurg Pediatr 7 : 643-649, 2011

7. Bouras T, Sgouros S: Complications of endoscopic third ventriculostomy. World Neurosurg 79(Suppl) : S22.e9-e12, 2013

8. Chong S, Lee JY, Kim SK, Phi JH, Wang KC : Individualized ventricular access using multi-plane brain images. Childs Nerv Syst $27: 299-302$ 2011

9. Cinalli G, Sainte-Rose C, Chumas P, Zerah M, Brunelle F, Lot G, et al. : Failure of third ventriculostomy in the treatment of aqueductal stenosis in children. J Neurosurg $90: 448-454,1999$

10. Cinalli G, Maixner W, Sainte-Rose C : Pediatric Hydrocephalus. Milano : Springer Science \& Business Media, 2012, pp138-140
11. de Kunder SL, Ter Laak-Poort MP, Nicolai J, Vles JS, Cornips EM : Fever after intraventricular neuroendoscopic procedures in children. Childs Nerv Syst 32 : 1049-1055, 2016

12. Grand W, Leonardo J, Chamczuk AJ, Korus AJ : Endoscopic third ventriculostomy in 250 adults with hydrocephalus: Patient selection, outcomes, and complications. Neurosurgery 78 : 109-119, 2016

13. Grunert P, Charalampaki P, Hopf N, Filippi R : The role of third ventriculostomy in the management of obstructive hydrocephalus. Minim Invasive Neurosurg $46: 16-21,2003$

14. Handler $M H$, Abbott $R$, Lee $M:$ A near-fatal complication of endoscopic third ventriculostomy: Case report. Neurosurgery 35 : 525-527; discussion 527-528, 1994

15. Hayashi N, Endo S, Hamada H, Shibata T, Fukuda O, Takaku A : Role of preoperative midsagittal magnetic resonance imaging in endoscopic third ventriculostomy. Minim Invasive Neurosurg 42 : 79-82, 1999

16. Hopf NJ, Grunert P, Fries G, Resch KD, Perneczky A : Endoscopic third ventriculostomy: outcome analysis of 100 consecutive procedures. Neurosurgery 44 : 795-804; discussion 804-806, 1999

17. Hwang SW, Al-Shamy G, Whitehead WE, Curry DJ, Dauser R, Luerssen $\mathrm{TG}$, et al. : Amenorrhea complicating endoscopic third ventriculostomy in the pediatric age group. J Neurosurg Pediatr 8 : 325-328, 2011

18. Isaacs AM, Bezchlibnyk YB, Yong H, Koshy D, Urbaneja G, Hader WJ, et al. : Endoscopic third ventriculostomy for treatment of adult hydrocephalus: long-term follow-up of 163 patients. Neurosurg Focus 41 : E3, 2016

19. Kawsar KA, Haque MR, Chowdhury FH : Avoidance and management of perioperative complications of endoscopic third ventriculostomy: the Dhaka experience. J Neurosurg 123 : 1414-1419, 2015

20. Kim IY, Jung S, Moon KS, Jung TY, Kang SS : Neuronavigation-guided endoscopic surgery for pineal tumors with hydrocephalus. Minim Invasive Neurosurg 47 : 365-368, 2004

21. Kinoshita Y, Tominaga A, Saitoh T, Usui S, Takayasu T, Arita K, et al. : Postoperative fever specific to neuroendoscopic procedures. Neurosurg Rev, 2013 [Epub ahead of print]

22. Kulkarni AV, Riva-Cambrin J, Holubkov R, Browd SR, Cochrane DD, Drake JM, et al. : Endoscopic third ventriculostomy in children: Prospective, multicenter results from the hydrocephalus clinical research network. J Neurosurg Pediatr 18 : 423-429, 2016

23. Naidich TP, Pudlowski RM, Naidich JB : Computed tomographic signs of the chiari ii malformation. III : Ventricles and cisterns. Radiology 134 : 657-663, 1980

24. Roth J, Ram Z, Constantini S : Endoscopic considerations treating hydrocephalus caused by basal ganglia and large thalamic tumors. Surg Neurol Int $6: 56,2015$

25. Schroeder HW, Niendorf WR, Gaab MR : Complications of endoscopic third ventriculostomy. J Neurosurg 96 : 1032-1040, 2002

26. Tafuri KS, Wilson TA : Growth hormone deficiency and diabetes insipidus as a complication of endoscopic third ventriculostomy. J Clin Res Pediatr Endocrinol 4 : 216-219, 2012

27. Teo $C$, Jones $R$ : Management of hydrocephalus by endoscopic third ventriculostomy in patients with myelomeningocele. Pediatr Neurosurg 
J Korean Neurosurg Soc 60 | May 2017

25 : 57-63; discussion 63, 1996

28. Wang KC, Cho BK, Kim CS, Kim SD : Control of intraoperative bleeding with hydrostatic pressure during endoscopic surgery. Childs Nerv Syst

$14: 280-284,1998$ 